\title{
Development of an X-ray Based Spectroscopy MicroXRF System with LA- ICP-MS Capabilities: Trace-Level Microns-scale Mapping and Femtogram Detection Sensitivity
}

$\underline{\text { SH Lau }}{ }^{1}$, Sylvia JY Lewis ${ }^{1}$, Wenbing Yun ${ }^{1}$, Benjamin Stripe ${ }^{1}$, Janos Kirz ${ }^{1}$, Alan Lyon ${ }^{1}$, David Reynolds $^{1}$, Richard Ian Spink ${ }^{1}$

1. Sigray, Inc. Concord, CA USA

In recent years, there have been significant advances in laser ablation inductively coupled mass spectrometry (LA-ICP-MS) toward improved resolution and increased sensitivity [1]. However, there are still known challenges in using the technique, including sample preparation, lack of matrix-matched standards needed for quantification, and artifacts such as elemental fractionation and polymeric interference [2].

We will present our patented AttoMap ${ }^{\mathrm{TM}}$ micro X-ray fluorescence (microXRF) system that features an innovative microstructured $x$-ray source and high efficiency $x$-ray optic combination to enable $<8 \mu \mathrm{m}$ resolution and sub-femtogram sensitivity. Micro x-ray fluorescence (microXRF) is a powerful technique that originated at the synchrotron and has demonstrated outstanding capabilities at the synchrotron, including down to sub-ppb detection sensitivity and submicron resolution. However, the capabilities of laboratory-based approaches to microXRF have been limited, primarily due to the low brightness of laboratory x-ray sources and the low efficiency of laboratory x-ray optics.

The key enabling technology of the system is its x-ray source, which uses an x-ray target comprised of fine microstructured metal x-ray emitters embedded in a diamond substrate. The design of the target enables rapid thermal dissipation, provided by the high degree of contact between the microstructures and diamond (which has excellent thermal properties) so that the diamond remains cool under high power loading, and the linear accumulation of x-rays at nearzero take-off angles through the largely $x$-ray transparent diamond. Used in combination with the source is an advanced $x$-ray mirror lens, developed from a high precision fabrication process capable of producing lenses with minimal slope errors and surface smoothness on the order of single digit angstroms.

The system's performance will be demonstrated with analysis of standards and results its application to various research applications. Applications of the system include: distribution analysis of nanoparticles down to $50 \mathrm{~nm}$, trace elemental mapping of metals in biological tissue in the biomedical field of metallomics, geochemical mapping, and advanced materials analysis.

[1] Butler et al. Journal of Analytical Atomic Spectrometry (2016).

[2] Limbeck et al. Analytical and Bioanalytical Chemistry (2015).

[3] The authors acknowledge funding from the NSF, Division of Industrial Innovation \& Partnerships for the development of x-ray mirror lens (IIP-1448727) and the NIH, National Institute of General Medicine Science for the development of the microstructured source target (GRANT11545218). 


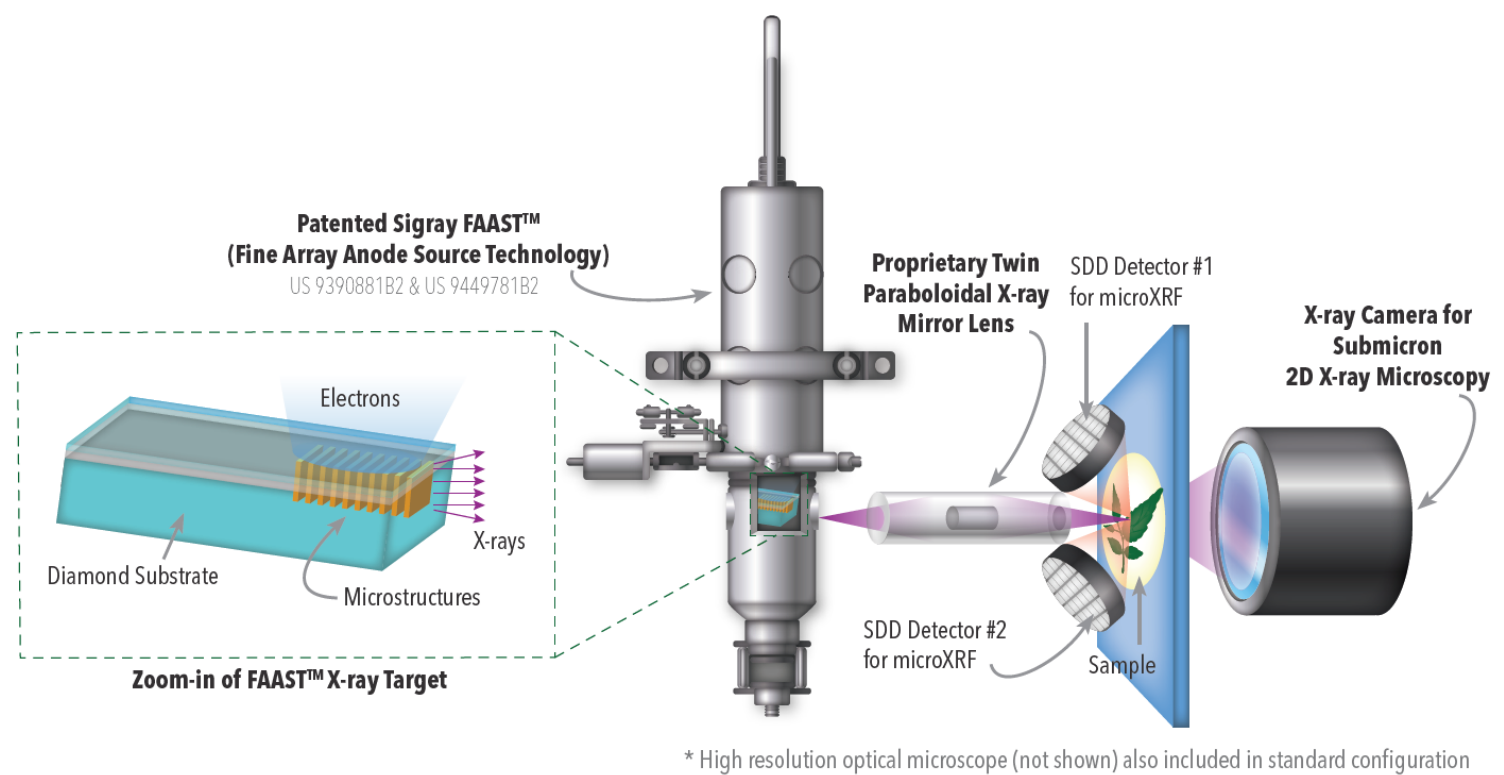

Figure 1. Patented technology within the AttoMap ${ }^{\mathrm{TM}}$ : microstructured $\mathrm{x}$-ray source, a high efficiency x-ray mirror lens produced through a proprietary manufacturing process, and two SDDs placed at optimized collection angles

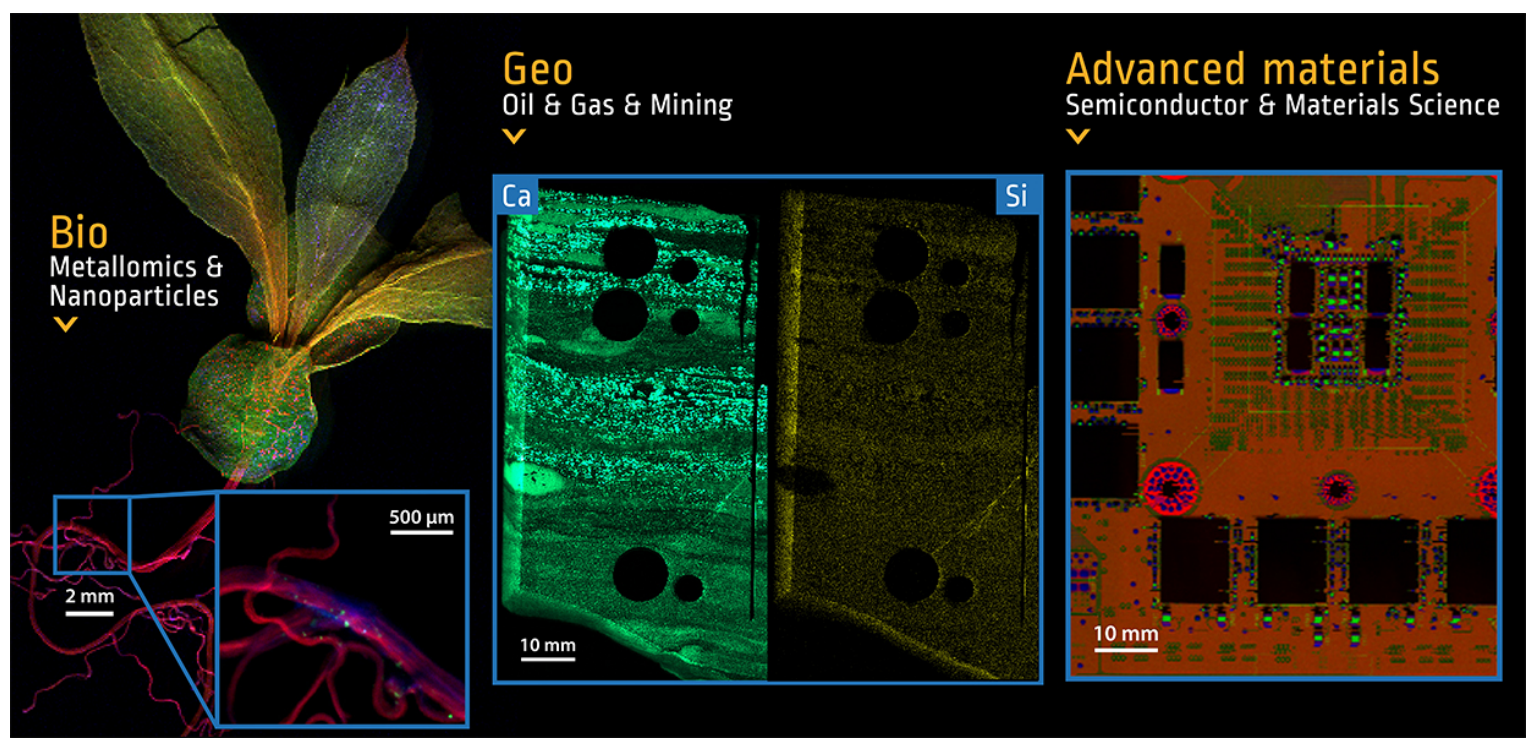

Figure 2. Exemplary applications of the AttoMap 